\section{REVISTA BRASILEIRA DE QUALIDADE DE VIDA}

\title{
Avaliação da percepção da qualidade de vida de jovens universitários: comparativo entre graduandos do turno diurno e noturno
}

\section{Evaluation of the quality of life of young college students: comparison between day time night time students}

\author{
Ana Claudia Petrini \\ Universidade Metodista de Piracicaba - UNIMEP - Piracicaba - Brasil \\ anaclaudia.petrini@yahoo.com.br \\ Gabriela Margato \\ Universidade Metodista de Piracicaba - UNIMEP - Piracicaba - Brasil \\ gmargato@gmail.com \\ Guanis de Barros Vilela Junior \\ Universidade Metodista de Piracicaba - UNIMEP - Piracicaba - Brasil \\ Faculdades Metropolitanas de Campinas - METROCAMP - Campinas - Brasil \\ guanis@gmail.com
}

\section{RESUMO}

OBJETIVO: O presente estudo teve como objetivo avaliar a percepção da qualidade de vida (QV) de jovens universitários, acadêmicos das áreas de ciências humanas e da saúde, buscando verificar se há diferenças na percepção da QV entre os graduandos dos turnos diurno e noturno.

MÉTODOS: Estudo observacional, transversal e descritivo. A amostra foi constituída por 62 universitários, jovens, graduandos de ambos os turnos, subdivididos em dois grupos iguais: grupo do turno diurno e grupo do turno noturno. Para a avaliação da QV optou-se pela aplicação do questionário WHOQOL-bref, desenvolvido pela Organização Mundial da Saúde (OMS). Para a obtenção dos resultados realizou-se a sintaxe do questionário WHOQOL-bref; o teste estatístico de Mann-Whitney foi aplicado para a comparação entre os grupos e a correlação de Spearman realizada entre os domínios do questionário inter e intragrupos.

RESULTADOS: Os domínios do WHOQOL-bref referentes à percepção da QV com escores maiores foram o domínio físico e o domínio das relações sociais, enquanto os domínios com escores menores foram o domínio psicológico e o domínio meio ambiente. As correlações foram significativas entre todos os domínios intragrupo, mas não existentes intergrupos.

CONCLUSÕES: A percepção da QV foi semelhante entre os grupos. Os resultados indicam a necessidade de atenção e melhoria, principalmente no que diz respeito aos aspectos inerentes aos domínios psicológico e ambiental.

PALAVRAS-CHAVE: Qualidade de vida. Universitários. WHOQOL-bref. 


\section{ABSTRACT}

OBJECTIVE: This present study has had as a main goal to assess the perception of the quality of life (QOL) of young college students, scholars from the humanities and health areas, aiming at verifying whether there are differences in the perception of QOL between day time and night time scholars.

METHODS: An observational, cross-sectional and descriptive. The sample consisted of 62 university, young, students of both rounds, divided equally into two groups, with 31 students from each term in each group. For the evaluation of the QOL, the application of the WHOQOL-bref questionnaire, developed by the World Health Organization (WHO), was chosen. In order to obtain the results, the syntax of the WHOQOL-bref questionnaire was conducted, as well as the MannWhitney signed-rank test for the comparison of the results between the groups and the Spearman's rank correlation coefficient among the domains of the inter and intra groups questionnaire.

RESULTS: There haven't been any significant statistical differences in the perception of QOL between the students of different terms; in both groups, the WHOQOL-bref domains with the highest scores have been the physical health and social relationships domains, whereas the ones with the lowest scores have been the psychological and environment domains. The correlations have been significant among all the intra group domains, but not the inter group ones.

CONCLUSIONS: The perception of QOL was similar between groups. The results indicate the need for attention and improvement, especially with regard to aspects related to the psychological and environmental.

KEYWORDS: Quality of life. College students. WHOQOL-bref.

\section{Introdução}

Atualmente, o constructo de saúde vem sendo discutido amplamente, com o propósito de correlacionar o termo citado à qualidade de vida (QV) dos indivíduos com suas características sociais, psicológicas e ambientais (FLECK, 2000; NAHAS, 2003).

Benjamin (1994) revelou que, desde a década de 80, o tema QV de estudantes universitários vem sendo pesquisado, e segue, com enfoque tanto objetivo como subjetivo. $O$ primeiro diz respeito ao grau de satisfação das necessidades do estudante, em função do seu desenvolvimento socioeconômico, já o segundo, diz respeito ao bem-estar do sujeito, bem como, sua percepção do nível de realização pessoal em âmbitos individuais e coletivos (OLIVEIRA; CIAMPONE, 2008).

Cunha e Carrilho (2005) salientam que o ingresso do indivíduo no ensino superior é acompanhado por uma série de adaptações, desde aquelas relacionadas à nova rotina até as inerentes às expectativas acadêmicas. Contudo, novos comportamentos são frequentemente adotados pelos estudantes nessa fase, e esses, podem impactar positiva ou negativamente na QV do indivíduo.

Para compreender e avaliar a QV dos indivíduos em geral, a Organização Mundial da Saúde (OMS), desenvolveu por meio do World Health Organization Quality of Life, (O Grupo WHOQOL), o questionário WHOQOL-bref, o qual integra a avaliação da capacidade física, do bem-estar psicológico, das relações sociais e do contexto ambiental em que se insere o indivíduo. (THE WHOQOL GROUP, 1995; FLECK, 2000; HWANG et. al., 2003).

O conceito de QV foi então definido, de maneira unificada e transcultural, pela OMS, como sendo a "[...] percepção individual de sua posição na vida, no contexto da cultura e sistemas de valores nos quais vive e em relação aos seus objetivos, expectativas, padrões e preocupações" (THE WHOQOL GROUP, 1995, p. 1405).

Por outro lado, a QV dos Estudantes (QVE) vem sendo compreendida como sendo a "[...] percepção de satisfação e felicidade, por parte do estudante em relação a múltiplos domínios de vida 
à luz de fatores psicossociais e contextuais relevantes e estruturas de significados pessoais" (OLIVEIRA; CIAMPONE, 2008, p. 58).

Nahas (2003) defende que o estilo de vida pode afetar a QV das pessoas e, portanto, o sujeito deve preocupar-se em modificar hábitos cotidianos, quando necessário, na busca do bem-estar e principalmente conscientizar-se de seus benefícios para a saúde em todos os campos, incluindo as atividades acadêmicas.

Sabe-se que, cursos superiores são oferecidos nos turnos diurno, vespertino, noturno e em período integral, dependendo do curso e da instituição de ensino superior (IES) escolhida pelo estudante (SAUPE, 2002). Cada turno pode, por sua vez, apresentar diferentes características dentro de uma rotina acadêmica.

Desse modo, o presente estudo teve como objetivo avaliar a percepção da QV de jovens universitários, acadêmicos de uma mesma universidade, comparando os graduandos dos turnos, diurno e noturno. Posteriormente, o objetivo específico do trabalho foi verificar a existência de possíveis correlações entre a percepção da QV e os resultados obtidos em cada domínio do questionário WHOQOL-bref, intragrupo e intergrupos.

\section{Metodologia}

Trata-se de um estudo com delineamento observacional, descritivo e transversal. Participaram do estudo 62 voluntários, estudantes universitários, jovens, com idade compreendida entre 18 e 25 anos, saudáveis, de ambos os gêneros, acadêmicos do $2^{\circ}$ e $3^{\circ}$ ano de graduação das áreas de ciências humanas e ciências da saúde, dos turnos diurno e noturno, recrutados em uma universidade privada, localizada na cidade de Piracicaba - SP.

Os voluntários foram distribuídos em dois grupos: o primeiro composto por 31 graduandos do turno diurno, e o segundo por 31 graduandos do turno noturno.

O estudo foi submetido e aprovado pelo comitê de ética local, sob protocolo $\mathrm{n}^{\circ} 76 / 09$, sendo realizado como parte de um projeto de iniciação científica e vinculado a um projeto-mãe. Todos os participantes foram devidamente informados dos objetivos, procedimentos e análises dos mesmos, assinando o termo de consentimento livre e esclarecido (TCLE).

Para a avaliação da percepção da QV foi utilizado o questionário WHOQOL-bref da OMS, constituído por 26 questões, numa escala do tipo Likert, avaliando-se positiva e/ou negativamente à percepção da QV.

As perguntas do questionário são distribuídas em quatro domínios: D1 - domínio físico, D2 domínio psicológico, D3 - domínio social e D4 - domínio ambiental, em que se consideram os últimos 15 dias vividos pelos sujeitos. Cada domínio possui questões cujas alternativas variam numa intensidade de 1 a 5, na interpretação da sintaxe do questionário escores mais próximos de 100 pontos são sugestivos de uma melhor percepção da QV.

O domínio físico é elaborado com ênfase nas seguintes facetas: dor e desconforto, energia e fadiga, sono e repouso, mobilidade, atividades da vida cotidiana, dependência de medicação ou de tratamentos e capacidade de trabalho. O domínio psicológico focaliza as seguintes facetas: sentimentos positivos, pensar, aprender, memória e concentração, autoestima, imagem corporal e aparência, sentimentos negativos, espiritualidade, religião e crenças pessoais. O domínio das relações sociais aborda as facetas: relações pessoais, suporte (apoio) social, atividade sexual. O domínio meio ambiente abrange as facetas: segurança física e proteção, ambiente no lar, recursos financeiros, cuidados de saúde e sociais: disponibilidade e qualidade, oportunidade de adquirir novas informações e habilidades, participação e oportunidades de recreação/lazer, e ambiente físico (poluição, ruído, segurança pública, trânsito, clima e transporte) (THE WHOQOL GROUP, 1995).

A aplicação do questionário foi realizada na própria universidade, sendo respondido em encontro único e tendo todas as informações preenchidas pelos próprios voluntários. O tempo para o preenchimento completo do questionário variou entre 5 e 15 minutos.

Distribuiu-se um total de 66 questionários entre os graduandos, porém quatro deles foram descartados do estudo por não estarem completamente preenchidos. 
O tratamento estatístico foi procedido com auxílio do pacote 'SPSS, 17.0' conforme os objetivos do estudo. A verificação da consistência interna do questionário foi realizada pelo Coeficiente de Alfa de Cronbach ( $\alpha$ ), a fim de verificar a confiabilidade das respostas.

Foi realizada a sintaxe do WHOQOL-bref para obtenção dos resultados do questionário e o teste estatístico de Mann-Whitney foi aplicado para a comparação dos resultados entre os grupos.

Por fim, o Coeficiente de Spearman foi realizado para verificar a existência de correlação entre os resultados dos domínios do questionário, intra e intergrupos. Em todos os casos os níveis de significância foram pré-fixados em $5 \%(\mathrm{p}<0,05)$.

\section{Resultados}

A média de idade dos grupos foi de 20,89 $( \pm 2,83)$ anos para os graduandos do grupo do turno diurno e 21,45 $( \pm 1,85)$ anos para os graduandos do grupo do turno noturno.

Com relação ao gênero, o grupo do turno diurno $(n=31)$ apresentou 11 sujeitos do gênero feminino e 20 do gênero masculino. Já o grupo do turno noturno $(n=31)$ apresentou 24 sujeitos do gênero feminino e 7 do gênero masculino.

$\mathrm{O}$ valor da consistência interna das respostas do questionário WHOQOL-bref em sua forma global foi de $\alpha=0,85$ para o grupo do turno diurno e $\alpha=0,76$ para o grupo do turno noturno.

A Tabela 1 representa a pontuação em média e desvio padrão dos domínios do WHOQOLbref, e o valor de p do teste de Mann-Whitney, aplicado para a comparação entre os grupos.

Tabela 1 - Média, desvio padrão e valor de p dos escores dos domínios do questionário WHOQOL-bref dos grupos

\begin{tabular}{lccc}
\hline DOMÍNIOS & Diurno & Noturno & p valor \\
\hline Físico & $72,23 \pm 12,96$ & $76,72 \pm 10,94$ & 0.2942 \\
Psicológico & $67,74 \pm 13,52$ & $67,47 \pm 11,09$ & 0.7729 \\
Relações Sociais & $70,69 \pm 17,06$ & $72,04 \pm 17,55$ & 0.6172 \\
Meio Ambiente & $61,59 \pm 16,22$ & $66,43 \pm 11,99$ & 0.1952 \\
\hline \multicolumn{4}{c}{$\mathrm{p}<0,05$} \\
\end{tabular}

Os valores de $\mathrm{p}$ indicam que não há diferenças estatisticamente significativas entre os resultados dos grupos.

A correlação de Spearman demonstrou haver correlações significativas entre os domínios intragrupo, ou seja, entre os domínios de cada grupo. Entretanto, não foram verificadas correlações significativas intergrupos, ou seja, entre os domínios de um grupo e de outro.

A Tabela 2 representa as correlações do grupo do turno diurno. Nesse grupo, as correlações ocorreram respectivamente entre todos os domínios.

Tabela 2 - Correlações entre os domínios do questionário WHOQOL-bref para o grupo do turno diurno

\begin{tabular}{|c|c|c|c|c|}
\hline & Domínio Físico & Domínio Psicológico & Domínio Social & $\begin{array}{c}\text { Domínio meio } \\
\text { ambiente }\end{array}$ \\
\hline Domínio Físico & & $0,621 * *$ & $0,408^{*}$ & $0,819 * *$ \\
\hline Domínio Psicológico & $0,621 * *$ & & $0,431 *$ & $0,589 * *$ \\
\hline Domínio Social & $0,408^{*}$ & $0,431 *$ & & $0,551 * *$ \\
\hline $\begin{array}{l}\text { Domínio Meio } \\
\text { Ambiente }\end{array}$ & $0,819 * *$ & $0,589 * *$ & $0,551 * *$ & \\
\hline
\end{tabular}

Já para o grupo do turno noturno, as correlações ocorreram entre os domínios: físico e psicológico; físico e social; físico e meio ambiente e entre o psicológico e o social. As correlações do grupo do turno noturno estão expressas na Tabela 3. 
Tabela 3 - Correlações entre os domínios do questionário WHOQOL-bref para o grupo do turno noturno

\begin{tabular}{|c|c|c|c|c|}
\hline & Domínio Físico & Domínio Psicológico & Domínio Social & $\begin{array}{c}\text { Domínio Meio } \\
\text { Ambiente }\end{array}$ \\
\hline $\begin{array}{l}\text { Domínio Físico } \\
\text { Domínio Psicológico } \\
\text { Domínio Social } \\
\text { Domínio Meio } \\
\text { Ambiente }\end{array}$ & $\begin{array}{c}0,554 * * \\
0,506 * * \\
0,379 *\end{array}$ & $\begin{array}{l}0,554^{* *} \\
0,670^{* *}\end{array}$ & $\begin{array}{l}0,506 * * \\
0,670 * *\end{array}$ & $0,379^{*}$ \\
\hline
\end{tabular}

\section{Discussão}

Com base nos resultados observados, o presente estudo indicou não haver diferenças na percepção da QV entre os jovens universitários frequentadores do turno diurno e noturno. Ao considerar-se que o valor mínimo aceitável para o coeficiente de Alfa de Cronbach se estabelece em 0,70 e o máximo em 0,90 (STREINER, 2003), os resultados adquiridos garantem confiabilidade satisfatória das respostas.

No que diz respeito ao domínio físico da QV, os jovens pesquisados, de ambos os turnos, demarcam uma percepção positiva, corroborando com o estudo de Catunda e Ruiz (2008), realizado com graduandos dos cursos de Educação Física, Psicologia e Sistemas de Informação, e Dias et. al. (2010), que avaliaram a QV de 100 alunos do curso de medicina de Sorocaba - PUC/SP, em que, os resultados apontam a avaliação positiva dos universitários, obtendo escores próximos a 100 pontos.

A percepção positiva do domínio físico demonstra que os graduandos pesquisados encontram-se satisfeitos com suas condições físicas, fato que pode estar relacionado com a capacidade íntegra de realização das tarefas cotidianas e corriqueiras dos universitários. Deve-se considerar que nos dias atuais são muitos aqueles que exercem inúmeras atividades diárias, além dos estudos (MANZATO et al., 2011), para tanto, a habilidade em realizá-las sem que haja algum tipo de desconforto e/ou dificuldade, contribui para a percepção positiva desse domínio.

O domínio psicológico obteve, em ambos os grupos, um escore menor se comparado ao domínio físico. Tal resultado vem ao encontro dos obtidos por Cunha et al. (2005), em uma pesquisa sobre a QV de graduandos do $1^{\circ}$ ano do curso de psicologia, e Catunda e Ruiz (2008), que também apontam a negatividade por parte dos universitários referente ao domínio psicológico.

A percepção relativa a esse domínio indica que os universitários, de ambos os turnos, podem estar insatisfeitos com as condições de vida pessoal, fato que, em algum momento da jornada acadêmica, pode refletir sobre a motivação do graduando. Como destacam Catunda e Ruiz (2008, p. 27):

\footnotetext{
Vale dizer que, se o aluno vivencia sentimentos negativos como mau humor, ansiedade e depressão (em sua vida particular e/ou como decorrência das próprias atividades acadêmicas) e, além (ou por causa) disso não conseguem se concentrar tão bem nos estudos, podendo ter cada vez mais prejudicada sua motivação para aprender e seu desempenho no curso escolhido. Ademais, os sentimentos negativos vivenciados podem comprometer sua saúde mental.
}

A pontuação do domínio das relações sociais aponta uma percepção positiva da QV aos grupos, vindo ao encontro dos resultados obtidos por Saupe (2002), que avaliaram a QV de acadêmicos de enfermagem, e aos de Manzatto et al. (2011), que realizaram uma pesquisa sobre o consumo de álcool e QV em estudantes universitários na cidade de Campinas-SP.

O resultado propõe que o convívio no meio acadêmico estimula a construção de novas amizades e intensifica o convívio social. A promoção da sociabilização no ambiente acadêmico é destacada por Manzatto et al. (2011), indicando que dentro do campo universitário, além do aprendizado, os estudantes buscam por um incremento na vida social. 
O domínio meio ambiente possui a menor pontuação diante dos quatro domínios do questionário, resultado observado também no estudo de Eurich e Kluthcovsky (2008), Manzatto et al. (2011) e Maciel et al. (2013).

Supõe-se que o escore baixo referente a esse domínio, tenha relação com possíveis implicações financeiras, que podem surgir defronte à rotina acadêmica. Maciel et al. (2013), em uma pesquisa realizada com uma comunidade universitária, concluíram que o domínio do meio ambiente apresenta associação com a renda, impactando na QV de indivíduos.

A qualidade dos transportes púbicos, bem como, os aspectos relacionados à segurança pública, também podem ter influências sobre o resultado obtido nesse domínio. De acordo com Vilela Junior et al. (2007), a ineficiência nos órgãos de segurança pública pode influenciar diretamente na QV dos indivíduos.

Com relação aos aspectos inerentes aos turnos, Barreiro e Terribili Filho (2007) enfatizam que, frente à realidade brasileira, sabe-se que os estudantes que trabalham durante o dia são aqueles que compõem a grande maioria dos estudantes do ensino superior noturno.

De acordo com Terribili Filho e Raphael (2005), os universitários do período noturno enfrentam maiores dificuldades inerentes à rotina acadêmica, e, podem ainda, não estar preferencialmente em sua área de interesse.

Pressupostos indicam que dificuldades, sobretudo no que se refere aos problemas com o trânsito e transporte, são encontradas pelos universitários do ensino superior noturno, e podem influenciar no índice de frequência as aulas, quando esses geram atraso ou perdas das mesmas (TERRIBILI FILHO; RAPHAEL, 2005).

Todavia, a limitação da presente pesquisa foi não obter informações complementares referentes às atividades de trabalho, atividades extra-acadêmicas e levantamento socioeconômico dos voluntários.

Ainda assim, defronte as características das áreas de formação dos voluntários, o fato da amostra se constituir de graduandos das áreas de ciências humanas e da saúde, pré-estabelece que os jovens possam estar constantemente envolvidos com estágios e/ou atividades práticas relativas às áreas.

Provavelmente seja esse um fator que favoreceu a semelhança encontrada entre os grupos com relação à percepção da QV. Além do mais, são jovens, acadêmicos de uma mesma universidade e uma mesma faixa etária, considerando-se a média da idade dos grupos.

No que diz respeito às correlações observadas nos domínios do WHOQOL-bref existentes somente intragrupo, é cabível expor que: a correlação entre o domínio físico e psicológico demonstra que o bem-estar físico fomenta o bem-estar psicológico; a correlação entre o domínio físico e domínio das relações sociais pressupõe que a convivência social pode ser favorecida pelo bem-estar físico; a correlação entre domínio físico e o domínio meio ambiente pondera que as condições do meio ambiente refletem no bem-estar físico dos indivíduos; a correlação entre o domínio psicológico e relações sociais transmite a ideia de que a interação social do universitário apresenta interferência em seu psicológico; já a correlação entre o domínio das relações sociais e o domínio do meio ambiente prescreve que as condições ambientais realçam as interações sociais.

Não foram verificadas correlações significativas entre os resultados dos domínios intergrupos. Dessa forma, revela-se que para que os grupos sejam semelhantes, não necessariamente exista uma correlação entre os seus domínios.

Embora não se tenha obtido excelência nos resultados dos domínios, a percepção da QV dos pesquisados é satisfatória para com os aspectos relacionados ao domínio físico e social, que foram os que mais contribuíram para a positividade da QV, sendo os aspectos relacionados ao domínio psicológico e meio ambiente os mais prejudicados e não satisfatórios aos graduandos.

Cunha e Carrilho (2005) enfatizam que o desempenho acadêmico dos graduandos esta relacionado com seu bem-estar físico e psicológico. Portanto, a IES deve priorizar o desenvolvimento global dos alunos e elaborar, com frequência, propostas preventivas que assegurem o sucesso acadêmico. 
Ainda, conforme Eurich e Kluthcovsky (2008) dentre os fenômenos que integram à QV de estudantes universitários, destacam-se a importância da IES e dos docentes, haja vista, a inestimável contribuição que concedem aos universitários em seu desenvolvimento ao longo da jornada acadêmica.

\section{Considerações finais}

Em relação à percepção da $\mathrm{QV}$, os resultados revelam que há uma percepção satisfatória da QV, com escores positivos nos domínios físicos e sociais. Entretanto, os escores referentes aos níveis ambientais e psicológicos são percebidos como negativos pelos jovens, independente do turno que frequentam as aulas, diurno ou noturno.

Os resultados obtidos ponderam a necessidade de atenção e melhoria no que concerne aos aspectos psicológicos e ambientais, que foram os mais prejudicados. Pode-se inferir, ainda, que ajustes na adoção de medidas que se voltem diretamente a QV dos universitários possam ser benéficos e relevantes para a integridade e sucesso acadêmico dos graduandos.

Sugere-se que outras pesquisas entre estudantes universitários, graduandos de diferentes turnos sejam realizadas, com maior número de sujeitos e caracterização da amostra.

\section{Referências}

BARREIRO, I. M. de F.; TERRIBILI FILHO, A. Educação superior no período noturno no Brasil: políticas, intenções e omissões. Ensaio: Avaliação e Políticas Públicas em Educação, v. 15, n. 54, p. 81-102, jan./mar. 2007. Disponível em: 〈http://www.scielo.br/pdf/ensaio/v15n54/a06v1554.pdf > Acesso em: 08 dez. 2011.

BENJAMIN, M. The quality of student life: toward a coherent conceptualization. Indicators, v. 31, p. 205-264, 1994.

CATUNDA, M. A. P.; RUIZ, V. M. Qualidade de vida de universitários. Pensamento Plural: Revista Científica, São João da Boa Vista, v. 2, n. 1, p. 22-31, 2008. Disponível em: <http://www.fae.br/plural/Vol_2_n_1_2008/artigo_qualidadedevidadeuniversitarios.pdf>. Acesso em: 16 out. 2011.

CUNHA, F. S. et al. Qualidade de vida em alunos do $1^{\circ}$ ano do curso de psicologia. Revista da SPTM, v. 9, n. 1, p. 164-169, jul./dez. 2005. Disponível em: <http://www.sptm.org.br/revista/rev9_2/art6_9_2.pdf>. Acesso em: 16 out. 2011.

CUNHA, S. M.; CARRILLO, D. M. O processo de adaptação ao ensino superior e o rendimento acadêmico. Psicologia Escolar e Educacional, Campinas, v. 9, n. 2, p. 215-224, 2005. Disponível em: < http://www.scielo.br/pdf/pee/v9n2/v9n2a04.pdf> Acessado em: 16 out. 2011.

DIAS, J. C. R. et al. Qualidade de vida em cem alunos do curso de Medicina de Sorocaba PUC/SP. Revista Brasileira de Educação Médica, v. 34, n. 1, p. 116-123, 2010. Disponível em: < http://www.scielo.br/pdf/rbem/v34n1/a14v34n1.pdf > Acessado em: 16 out. 2011.

EURICH, R. B.; KLUTHCOVSKY, A. C. G. C. Avaliação da qualidade de vida de acadêmicos de graduação em Enfermagem do primeiro e quarto anos: influência das variáveis sociodemográfica. Revista de Psiquiatria, v. 30, n. 3, p. 211-220, 2008. Disponível em: <http://www.scielo.br/pdf/rprs/v30n3/v30n3a10.pdf >. Acesso em: 16 out. 2011.

FLECK, M. Aplicação da versão em português do instrumento abreviado de avaliação de qualidade de vida 'WHOQOL-bref'. Revista de Saúde Pública, v. 34, n. 2, p. 178-183, 2000. 
HWANG, H.; LIANG, W; CHIU, Y.; LIN, M. Suitability of the WHOQOL-bref for communitydwelling older people in Taiwan. Age Ageing, v. 32, n. 6, p. 593-600, 2003.

MACIEL, E. S. et al. Correlação entre nível de renda e os domínios da qualidade de vida de população universitária brasileira. Revista Brasileira de Qualidade de Vida, v. 05, n. 01, p. 53-62, 2013. Disponível em: < http://revistas.utfpr.edu.br/pg/index.php/rbqv/article/view/1458/957> Acessado em: 20 jun. 2013.

MANZATO, L. et al. Consumo de álcool e qualidade de vida em estudantes universitários. Revista da Faculdade de Educação Física da Unicamp, v. 9, n. 1, p. 37-53, jan./abr. 2011. Disponível em: < http://fefnet178.fef.unicamp.br/ojs/index.php/fef/article/view/528 > Acessado em: 08 ago. 2011.

NAHAS, M. V. Atividade física, saúde e qualidade de vida: conceitos e sugestões para um estilo de vida ativo. 3. ed. Londrina: Midiograf, 2003.

OLIVEIRA, R. A.; CIAMPONE, M. H. T. Qualidade de vida de estudantes de enfermagem: a construção de um processo e intervenções. Revista da Escola de Enfermagem da USP, v. 42, n. 1, p. 57-65, 2008. Disponível em: <http://www.scielo.br/pdf/reeusp/v42n1/08.pdf>. Acesso em: 07 jun. 2011.

SAUPE, R. Qualidade de vida de estudantes de Enfermagem conforme escala de Flanagan. Ciência, Cuidado e Saúde, v. 1, n. 2, p. 287-291, jul./dez. 2002.

STREINER, D. L. Being inconsistent about consistency: when coefficient alpha does and doesn't matter. Journal of Personality Assessment, v. 80, p. 217-222, 2003. Disponível em: <http://www.ncbi.nlm.nih.gov/pubmed/12763696> Acessado em: 16 jul. 2011.

THE WHOQOL GROUP (1995). The World Health Organization Quality of Life Assessment (WHOQOL): Position Paper from the World Health Organization. Sociais Ciência e Medicina, v. 41, p. 1403-1409, 1995.

TERRIBILI FILHO, A.; RAPHAEL, H. S. Fatores de atrasos e faltas do estudante do ensino superior noturno: a perda de aulas, de provas e o impacto no seu aproveitamento e em avaliações. Avaliação, Campinas, v. 10, n. 2, p. 117-135, 2005.

VILELA JUNIOR, G. B. et al. Comparação da qualidade de vida de mulheres idosas praticantes e não praticantes de exercício físico. Lecturas Educación Física y Deportes, v. 11, n. 106, p. 1-2, 2007. 